\title{
Editorial
}

Herz 2015 · 40:741

DOI 10.1007/s00059-015-4337-9

Online publiziert: 2. Juli 2015

(c) Urban \& Vogel 2015

Ralf Zahn

Medizinische Klinik B, Klinikum der Stadt Ludwigshafen am Rhein gGmbH, Ludwigshafen, Deutschland

\section{Interventionelle Therapie struktureller Herzerkrankungen}

Prof. Sievert, Frankfurt, die Möglichkeiten und klinischen Daten zum interventionellen Verschluss eines persistierenden offenen Foramen ovale (pFO) bei Patienten mit Zustand nach Schlaganfall sowie von Vorhof- (ASD) und Ventrikelseptumdefekten (VSD) dar. Während der pFO- und der Vorhofseptumverschluss technisch einfacher sind und in nennenswerten Fallzahlen vorkommen, ist der interventionelle VSD-Verschluss technisch sehr anspruchsvoll und kommt zudem eher selten vor. Im letzten Beitrag stellt Herr Dr. Werner aus Ludwigshafen die interventionellen Möglichkeiten zum Verschluss paravalvulärer Leckagen nach operativem oder interventionellem Klappenersatz vor. Auch hier hat sich der interventionelle Ansatz mit der Implantation von sog. "plugs“ (Stopfen), der auch technisch sehr herausfordernd ist, als eine wichtige Ergänzung oder gar als Mittel der Wahl gegenüber einer Reoperation etabliert.

Insgesamt sind alle diese behandelnden Prozeduren technisch anspruchsvoll und bedürfen neben eines in diesen Techniken geübten interventionellen Kardiologen ganz besonders der sorgfältigen Indikationsstellung, die idealerweise in einem „Herzteam“, bestehend zumindest aus je einem Kardiologen und Herzchirurgen, getroffen werden sollte.

\section{Korrespondenzadresse}

Prof. Dr. R. Zahn

Medizinische Klinik B

Klinikum der Stadt Ludwigshafen am Rhein

gGmbH, Bremserstraße 79

67063 Ludwigshafen

erzahn@aol.com

\section{Literatur}

1. Vahanian A, Alfieri O, Andreotti F et al (2012) Guidelines on the management of valvular heart disease (version 2012): the Joint Task Force on the Management of Valvular Heart Disease of the European Society of Cardiology (ESC) and the European Association for Cardio-Thoracic Surgery (EACTS). Eur Heart J 33:2451-2496

2. Kuck KH, Eggebrecht H, Figulla HR et al (2015) Qualitätskriterien zur Durchführung der transvaskulären Aortenklappenimplantation (TAVI). Kardiologe 9:11-26 